\title{
INFOXICACIÓN: IMPLICACIONES DEL FENÓMENO EN LA PROFESIÓN PERIODÍSTICA
}

Ruth Franco Rodríguez ${ }^{\mathbf{1}}$ : Universidad Rey Juan Carlos. España. ruth.franco@ciberimaginario.es

Manuel Gértrudix Barrio: Universidad Rey Juan Carlos. España. manuel.gertrudix@urjc.es ORCID: 0000-0002-5869-3116

\section{RESUMEN}

La sobreabundancia informativa característica de la era digital plantea un desafío en la sociedad, principalmente para aquellos que, por su profesión, se ven obligados a lidiar con grandes cantidades de datos a diario, no pueden ignorar la información de entrada, y son los encargados de otorgar valor a la información en una sociedad cada vez más saturada por el volumen de la misma. La investigación analiza el impacto e incidencia de la infoxicación en los profesionales recién formados en periodismo. Mediante grupos focales, se evalúan los vectores que permiten comprender la complejidad del fenómeno y su repercusión en el modo de generar contenidos. Los resultados ofrecen una relación causal entre cantidad, desconfianza y saturación informativa, así como un deterioro de la capacidad de concentración ocasionado por el uso intensivo de internet, especialmente en dispositivos móviles.

PALABRAS CLAVE: Infoxicación - nativos digitales - periodismo - economía de la atención - internet - adicción tecnológica - dieta informativa

\footnotetext{
${ }^{1}$ Ruth Franco Rodríguez: colaboradora del Grupo de investigación Ciberimaginario de la Universidad Rey Juan Carlos y autora de la investigación "Sobreabundancia informativa y economía de la atención".
}

Correo: ruth.franco@ciberimaginario.es 


\title{
INFOXICATION: IMPLICATIONS OF THE PHENOMENON IN JOURNALISM
}

\begin{abstract}
Information overload, which is a characteristic of the digital age, is a societychallenge, especially for journalists, people whose jobs are daily forced to deal with a large amount of data, and they can't ignore the input-information. So, they are responsible ofaddingvalue to information in an increasingly saturated world by its volume. This research analyzes the impact and incidence of infoxication in journalists. Through focus groups, vectors are evaluated tounderstand the complexity of this phenomenon and to determine the impact on the way people create articles on the Internet. The results provide a causal relationship between quantity, distrust and information overload, as well as deterioration at the concentration ability, due to the intensive use of internet, especially from mobile devices.
\end{abstract}

KEYWORDS: Information overload - digital natives - journalism - attention economy internet - technology addiction - information diet

\section{INTRODUCCIÓN}

Con la llegada de la era digital, los profesionales de la comunicación, los publicistas $\mathrm{y}$, sobre todo, los lectores, que antes eran receptores pasivos, han hallado en internet el medio para librarse de todo tipo de restricciones, pudiendo generar y difundir contenidos con total libertad. Ante esta situación, el volumen de datos se ha ido incrementado exponencialmente en los últimos años. En 2002, la información almacenada en formato digital superaba al conjunto de datos analógicosgenerados hasta entonces (Lyman \& Varian, 2003). Cinco años más tarde, el $94 \%$ del total de la información se hallabaya en formato digital (Hilbert \& López, 2011), lo que demuestra que el ritmo de producción en internet es tan vertiginoso que resulta complicado de contabilizar. Y más cuando en tan solo un minuto se comparten de media unos 2.460 .000 contenidos en Facebook, se publican 277.00 nuevos tuits, se suben 216.000 fotos a Instagram o se añaden 72 horas de vídeo en YouTube. Estas cifras solo concierne a la actividad en redes sociales, pero el volumen crece todavía más fuera de ellas. Un ejemplo de ello son los 204 millones de emails que se estima que se envían de mediaen el mundo cada minuto que pasa(DOMO, 2014).

Mientras millones de terabytes se almacenan de forma apresurada en la nube, el tiempo para atenderlos disminuye progresivamente. Esto evidencia un deterioro, en este contexto, de la denominada economía de la atención(Goldhaber, 2006). El sobreesfuerzo mental que supone asimilar más datos de los que permite la capacidad humanaconduce al fenómeno de la infoxicación(Cornella, 2000), también conocido como Síndrome de fatiga informativa (Lewis, 1996). 
Son diversos los estudios que se han realizado sobre la sobrecarga cognitiva y todos ellos apuntan en la misma dirección. El académico de Ciencia CognitivaDavid Kirsh identifica una condición de saturación cuando a la sobreabundancia informativa se le añaden la ejecución de varias tareas y las interrupciones. El multitasking ha producido un salto cognitivo que posibilita ejecutar varios procesos simultáneamente, pero deteriora la capacidad de concentración porque ninguna tarea se asociaúnicamente a una zona del cerebro, sino que se trata de una red de redes y puede requerir actividad también en la zona cortical de la otra, por lo que se interrumpe el procesamiento de ambas (Kirsh, 2000).

Si se tiene en cuenta la Teoría de redes atencionales (Posner \& Petersen, 1990), la infoxicación repercutiría directamente en el sistema atencional anterior, encargado de realizar un esfuerzo mental para que el sujeto se concentreen un determinado estímulo. Los síntomas de una lesión en este área del cerebro se asemejan a los que una persona con déficit atencional padece: distractibilidad o trastorno de concentración. Es decir, incapacidad para atender, procesar en paralelo y dificultad para tomar decisiones (Klingberg, 2009).

\subsection{Sociedad hiperconectada}

En 2014 el número de hogares en España con acceso a internet representaba el $74,4 \%$ del total de la población, y por primera vez el principal tipo de conexión fuela conexión por banda ancha móvil(INE, 2014). Esta tendencia refleja que los smartphones tienen más penetración en la vida de las personas, tanto es así que se les dedica más tiempo del que se desearía en actividades cotidianas. Según un estudio de Nielsen(2014), se estima que los usuarios estadounidenses pasan de media unas 37 horas y 28 minutos al mes utilizando las diferentes apps instaladas en su dispositivo móvil; y que acuden al visionado de contenidos para distraerse mientras se encuentran en la consulta del médico o en la cola del supermercado aguardando su turno(Nielsen, 2015).

Los datos pueden aplicarse al ámbito nacional. El barómetro de marzo del CIS(2015) revelaque del $92,9 \%$ de los españoles que utiliza el teléfono móvil, el 39,6\% lo hace continuamente $y$, otro tanto, también el $39,6 \%$, lo hace varias veces al día. Al impulso repentino y casi involuntario de revisarconstantementeel móvil tras la alerta de la luz intermitente se le denomina nomofobia (no-mobile-phone-phobia)(The App Date, 2013). Este fenómeno afecta principalmente a jóvenes. Un estudio perteneciente al Centro Reina Sofía sobre Adolescencia y Juventud refleja que la mitad de los españoles de entre 16 a 20 se sienten con frecuencia saturados por el uso de las TIC, hasta el punto de necesitar desconectarse de ellas. Y el $85 \%$ de los encuestados declaró que la gente de su edad depende bastante o mucho de las redes sociales, y que el tiempo que pasan en internet es excesivo(Ballesteros Guerra \& Megías Quirós, 2015) Los datos se sitúan en la misma línea que la citada encuesta 
del CIS(2015), que desvela también que un $51,8 \%$ de la población española cree que pierde el tiempo con las TIC, y que casi el $62 \%$ considera que con las nuevas tecnologías el tiempo que se dedica a leer libros, periódicos o revistas en papel es cada vez menor.

\subsection{Enfoques teóricos}

Los cambios en los hábitos de consumo pueden explicarse desde la Teoría del medio de Marshall McLuhan (1993), en la cual se postula que el medio modifica la percepción y la comprensión del sujeto, así como la lectura fragmentada y distraída se sustenta en la Teoría del hipertexto en relación con la Teoría de juegos, pues el hipertexto explica los diferentes enlaces -recorridos o jugadas- que el usuario puede emprender en su lectura (Díaz Noci, 2004). De este modo, y teniendo en cuenta la teoría sociocultural(Harris, 1990), en la que el origen de la transformación social se sitúa en la innovación tecnológica, podría decirse que internet y las nuevas tecnologías estarían actuando comoactores de un nuevo escenario informacional, donde el procesamiento en paralelo y la multitarea configuran el entramado social.

Otro enfoque teórico que permitiría explicar la ingente cantidad de información disponible en internetes la ausencia del gatekeeper(White, 1950), o del portero de la información, ya que el volumen de datos a los que se tiene acceso ahora es tan elevado y proviene de tal cantidad de fuentes -profesionales de la información, expertos en determinadas áreas y usuarios que tienen blogs o páginas webs- que resulta complicado regular su flujo.Asimismo, la agenda mediática, entendida como los temas que los medios dan cobertura por ser transcendentes para la sociedad (McCombs, 1996), se desvirtúa en beneficio de una dieta informativa personalizada, que atiende cada vez más a los intereses de cada individuo.

\subsection{Desafíos en la profesión periodística}

El periodismo ha pasado por un proceso de adaptación del modelo informativo a los nuevos soportes donde los profesionales de la comunicación han hallado más acceso a la información, como consecuencia de la llegada de una red caracterizada por la ausencia de regulación, que la hace más abierta y libre, por iniciativas relacionadas con el periodismo de datos y la participación ciudadana, o por el simple hecho de disponer de nuevas herramientas con las que recabar información al instante, como los microbloggins o el uso de wikis para documentarse.

Según el informe La Función de comunicación vista por los profesionales de la información de AxiCom(2013), un periodista suele recibir de media al día entre 100 y 200 notas de prensa, propuestas de entrevistas y reportajes. El hecho de leer y valorar de manera razonable el valor informativo que se le otorga, tanto a la nota de 
prensa como a la propuesta, exige dedicarle como mínimo cinco minutos. Si esto se multiplica por el cómputo total de notas y propuestas que recibe al día, supondría invertir un tiempo imposible de afrontar, puesto que debe ocuparse también de hacer un seguimiento de los temas actuales y escribir sobre tales sucesos (AxiCom y Top Comunicación, 2013).No hay que olvidar que esta situación de sobreabundancia informativa se presenta en un contexto de precariedad laboral que dificulta producir y presentar un producto de calidad. Según el informe de la Asociación de la Prensa de Madrid (APM), desde el comienzo de la crisis se han destruido 11.875 empleos en España, además de una reducción salarial que ha ido en aumento(APM, 2014). Ante esta situación, caracterizada por recurrentes EREs, escasez de personal y mismo o más volumen de trabajo en menos manos, percibir infoxicación es casi inevitable.

\section{OBJETIVOS}

Los nativos digitales dedican gran parte de su tiempo a buscar información, realizar actividades en la red $y$, a menudo, creen tener la habilidad de ejecutar varias tareas a la vez. Si además estudian periodismo, una profesión que exige manejar grandes cantidades de datos a diario, estos sujetos se convierten en uno de los sectores de la población más expuestos al riesgo de la infoxicación. En este contexto, el objetivo general de la investigación se centra en analizar la visión que tienen los graduados en Periodismo recién insertados en el mercado laboral y los jóvenes estudiantes de esta titulación sobre cómo opera la infoxicación en su tarea informativa, tanto a nivel profesional como personal. Son objetivos específicos: a) identificar vectores que permitan comprender la complejidad del fenómeno, b) analizar el modo que tienen los sujetos de producir contenidos en la red, sobre todo los más ligados a la actualidad inminente, y c) evaluar en qué medida las herramientas de selección y filtrado contrarrestan sus efectos.

\section{METODOLOGÍA}

Para abordar el estudio se recurrió a un Focus Group: técnica cualitativa de obtención de datos permite recoger datos sustantivos sobre la visión que tienen los individuos sobre un determinado tema (Berg \& Lune, 2004).

La población objeto de estudio se acotó ajóvenes nacidos en las décadas ochenta o noventa del pasado siglo, considerando las siguientes variables sociodemográficas: edad, nivel de estudios y región. Se seleccionó una franja de edad comprendida de entre los 18 y 28 años, equivalente al segmento de nativos digitales(Prensky, 2010), con el fin de garantizar que los individuos estuviesen familiarizados con el uso de las TIC. El nivel de estudios se limitó a estudiantes universitarios y graduados en Periodismo, entendiendo que estos profesionales gestionan a diario un gran volumen de información. Por último, sesegmentó a nivel regional, incluyendo únicamente 
universidades públicas pertenecientes a la Comunidad de Madrid, por lo que los resultados y teorías generadas solo pueden considerarse válidas si se extrapolan a este segmento de la población.

El método de reclutamiento ejecutado en el Focus Group fue el mecanismoBola de nieve: localización inicial de participantes de diferentes círculos relacionados con el periodism para que, posteriormente, los segundos recurriesen a sus círculos y reclutasen a otros sujetos potenciales (Mella, 2000).

El trabajo de campo se realizó en Madrid el día 13 de diciembre de 2014 y fueron diez las personas que participaron en el estudio. Todas ellas procedían de las universidades Rey Juan Carlos, Carlos III y Complutense de Madrid. Por último, para el proceso de análisis se optó por seguir un método inductivo con la finalidad de generar nuevo conocimiento -en forma de teorías o discurso- a partir de las interpretaciones de las experiencias contadas por los sujetos. En virtud, se ha utilizado el método de la Teoría Fundamentada, pues permite recopilar datos y analizarlos de manera sistemática a través de categorías de abstracción. La recolección de datos se hizo mediante una transcripción completa. Su posterior análisis siguió las líneas generales de la codificación categórica -abierta, axial y selectiva- para después profundizar en los datos y reducirlos hasta hallar interpretaciones más abstractas que permitiesen la construcción final de la teoría o discurso emergente(Strauss, 2002).

\section{DISCUSIÓN}

\subsection{Resultados}

\subsubsection{Estrategias para informarse en internet}

Preguntados por el modo de informarse en internet ${ }^{2}$, los periodistas coincidieron en señalar la búsqueda, selección y contraste de fuentes como procesos clave para dar con la información deseada. Expresiones como "ir a la fuente original", "buscar las palabras clave", "saber cuáles son las fuentes verdaderas", "tener un buen filtro", "saber enfocar en algo concreto" y "seguir tu propio criterio" emergen continuamente a lo largo de la discusión.

Para evitar los problemas relacionados con el exceso de información y saturación, se observa una tendencia general a seleccionar y filtrar en función de la temática y la especialización de cada uno de los informantes. P4: "Queremos saberlo todo pero somos incapaces de abarcar tanta información. Si tú quieres saber algo sobre un tema, solo tienes que especializarte en ese tema". Los periodistas otorgan mayor

\footnotetext{
${ }^{2}$ Transcripción completa del Focus Group, disponible en:

(https://www.researchgate.net/publication/279449674_FOCUS_GROUP)
} 
relevancia a una información si está dentro de sus preferencias informativas, ya sea porque les afecta a nivel personal o en el ámbito laboral. Así, igual que uno debe informarse de aquello que le atañe directamente por sus intereses, también debe saber aplicar un criterio de selección en función de la propia especialización del medio. Algunos de los sujetos prefieren informarse a través de un medio especializado, si lo que están buscando es concreto, que en uno generalista por mucha reputación que tenga. Esto refleja que la fuente y la notoriedad son otro de los criterios a tener en cuenta por los sujetos a la hora de informarse. P01: "No es lo mismo leer un blog desconocido de una persona. $Y$ otra cosa, si es un periodista con nombre, da igual que escriba en 'El País' o que escriba en blogger, le van a leer y se van a fiar de su palabra".

Cabe destacar también que los informantes evidencian en la red un problema mayor a la hora de verificar la autenticidad de la información, por lo que consideran primordial extremar la desconfianza contrastando las fuentes y los contenidos publicados en distintos medios para obtener una significación más completa de un mismo suceso.

\subsubsection{Percepciones derivadas del exceso informacional}

Las sensaciones descritas por los sujetos objeto de estudio ante la cantidad de información que hay en la red se dedujo de expresiones que evidenciabanestados de ánimo negativos. Una de estas sensacioneses la desconfianza, experimentada por todos los sujetos, incluso cuando aseguraron consultar fuentes originales. Se percibe en la conversación con expresiones como "poner en duda todo", "el problema de la veracidad", "no puedes saber realmente lo que está pasando". Puede explicarse con la visión que da uno de los periodistas:

"A veces necesitas que una persona te diga 'yo lo sé y está pasando esto'. Porque como no sabes de quién fiarte y al final no te fías de nadie, pues no sabes muy bien qué información es fiable o qué información es mejor dejar de lado y al final es un lío todo" (P04).

Otra percepción que experimentan de manera indirecta, como consecuencia del volumen de información a la que tienen acceso en internet, es la insatisfacción. La sensación de fracaso o derrota avivada por el deseo de informarse más y mejor de lo que finalmente se consigue. Es, por tanto, el resultado de la saturación informativa y lo conforman expresiones como "la sensación de que no me lo he leído todo", "falta muchísimo para estar informados", "sensación de que no lo voy a conseguir", "creo que podría llegar a más y no llego".

En parte, este sentimiento está alimentada por la propia desconfianza que genera la procedencia de la fuente, no hallar la información que a uno le es útil y la falta de criterio personal y de filtrado, ya que si no existe un 'cuello de botella', la cantidad de 
información entrante para atender será mayor, y el proceso para informarse se complicará puesto que todo vale o tiene el mismo peso e interés. Este argumento se sustenta con las siguientes declaraciones: "Cuánto más tienes, más te agobias. Vas a leer menos y lo que leas, lo vas a leer peor"(P09);"Es como que te ayuda, pero no te hace sentir mejor informado"(Po8). Y justo cuando estos mecanismos fallan, la saturación, debido a la gran cantidad de estímulos que solicitan la atención del sujeto, crece y el resultado es experimentar sensaciones de agobio y preocupación.

\subsubsection{Déficit de atención vs concentración}

Los sujetos identifican un deterioro de su capacidad para atender a un único estímulo. Uno de los principales motivos por los que no pueden concentrarse se debe a las actividades simultáneas asimiladas como rutinasy que ponen en práctica a la hora de informarse. Algunas de las tareas descritas por los informantes son: "ver la televisión y leer al mismo tiempo", "leer y hablar a la vez a mi madre", "estudiar y escuchar música", "conducir y contestar a mensajes por el móvil", etc. La multitarea es un inconveniente mayor en el ámbito profesional, concretamente por su repercusión durante el proceso de documentación y redacción.

La distracción es otro de los motivos identificados por los informantes: "te desconcentras", "no te enteras de lo que estás leyendo", "te desvías y vuelves", "somos incapaces de quedarnos cierto tiempo centrados". Es el resultado de poner en práctica el multitasking. Entiéndase mejor con la siguiente cita, referida a la cantidad de páginas que los usuarios pueden tener abiertas a la vez en su navegador.

P04:"Estás leyendo una información en 'El País', por ejemplo. Me interesa, pero luego en relacionados veo otra que me quiero leer y pincho, y la miro por encima, aunque luego regrese a la otra y siga leyendo. Pero ya estoy con dos o tres a la vez".

Los principales 'distractores' que identifican los informantes en medios digitales son los enlaces que se incluyen dentro de las noticias, que redirigen la atención del sujeto a otra página con información complementaria -o no-, sobre el tema en cuestión; la propia portada, que puede convertirse en un enemigo cuando uno entra en un medio sin la idea fija de leerse una noticia en concreto. De hecho, el problema aparece cuando el sujeto desea leer varias noticias y termina abriendo en otras pestañas del navegador más contenidos que le interesan. Al igual les ocurre con el módulo de noticias relacionadas, ubicado normalmente en la esquina inferior de la pantalla, que termina desviando a los sujetos de la lectura prioritaria.

Otro factor, de mayor peso si cabe, que incide en esta cuestiónson las notificaciones procedentes de redes sociales y apps de mensajería instantánea, que se revisan 
desde el teléfono móvil. De hecho, califican al smartphone como el principal enemigo de su atención. Fue en ese momento cuando se identificó que la adicción tecnológica es uno de los pilares básicos del déficit de atención.Los sujetos contestan mensajes de WhatsApp y revisar notificaciones de Facebook y Twitter en su puesto de trabajo y/o en clase. Igualmente, reconocen usar el móvil más horas de las deseadas. "El móvil siempre está a tu lado", "si te escriben, contestas", "voy a la cocina y me llevo el móvil", "yo voy al baño con el móvil", "es la obsesión", "te imaginas que suena", detallan. Estas citas reflejan con claridad una relación de dependencia, que se refuerza con esta aportación:

"Nos hemos acostumbrado a nacer y a mamar internet y la tecnología en general. Entonces, estamos acostumbrados a integrarla en nuestra vida diaria de tal forma que todos los días tenemos que hacer uso de ella. De hecho, nos quedamos sin ella y no sabemos qué hacer" $(\mathrm{PO} 1)$.

\subsubsection{La inmediatez en la información periodística}

A medida que iba avanzando la discusión, emergieron cualidades de los medios digitales que repercuten en la producción y difusión informativa. Los sujetos perciben que la lucha de ser el primero en dar la noticia y aparecer en los resultados de búsqueda de Google tiene consecuencias contraproducentes en la profesión. Una de ellas la perciben durante el proceso de elaboración, pues aseguran consultar las fuentes en Twitter si se trata de un suceso de última hora y recurren a un formato timeline con informaciones muy escuetas. "Usar herramientas como Twitter", "ha pasado esto", "iremos actualizando más adelante", "un timeline con las actualizaciones", "viendo de agencia cuántos fallecidos iban habiendo" y "tienes que dar lo más importante" son algunas de las rutinas periodísticas asimiladas ante la urgencia del medio.

La inmediatez no solo afecta al proceso de elaboración, sino también al propio periodista que escribe el artículo bajo la presión del medio. Todos ellos, a la cuestión de cómo se sentían bajo la inmediatez, expresaron sentirse inseguros, bloqueados, frustrados y hasta decepcionados porque el producto presentado no iba a estar a la altura. Estos comentarios reflejan alguna de las sensaciones que experimentan habitualmente cuando se someten a un proceso de documentación y redacción con tan escaso tiempo: "Me bloqueo. Yo no puedo hacer una noticia en tan poco tiempo y sí lo hago, me rayaría mucho. Lo pensaría muchísimas veces, aunque leyera cinco segundos, no lo asimilaría" (P08);"Es que por mucho que te documentes, puede salirte una cosa a la que no están acostumbrado tus lectores. La culpa es tuya, pero a ti te están obligando a publicar en poco tiempo" (P05).

Por último, los informantes son conscientes de que este tipo de información genera una merma de calidad periodística y explicancómo perciben lalectura de estos productos informativos. 
"Luego eso se percibe como lector. Yo, vamos, de alguna vez que lees una noticia y..., que se nota que no está elaborada. Esa prisa, con faltas de ortografía, repeticiones" (p07); "En medios en los que he trabajado, lo que hacíamos era coger información de otros medios. Entonces, si la información de esos medios estaba mal, tú también metías la pata" (P09).

\subsubsection{Lectura lineal vs hipertextual}

En cuanto al modo de lectura, los periodistasoptraon por la lectura en papel porque es lineal y les permite concentrarse más. Así lo explicaban algunos de ellos:"Yo también prefiero leer en papel. Me gusta más. Si tengo que leer algo detenidamente, me gusta más en papel. Te concentras mejor. Me canso menos también"(P01);"Es mucho más cómodo. Lo tienes todo a mano. Estás centrado en la lectura. No te vas a otras páginas".Sin embargo, una minoría de los participantes prefería la lectura digital por las ventajas que ofrece en cuanto a ubicuidad y comodidad ( $\mathrm{PO} 10)$.

Sobre la lectura digital, la mayoría de los periodistas destacó la distracción asociada al carácter hipertextual de la web, por la posibilidad de dirigir la atención a otras partes de la pantalla.

Po:4 "Si ves un titular que te llama la atención, a lo mejor sí que ibas a buscar algo y paras en esa otra noticia. $\mathrm{O}$ si por ejemplo en Twitter estás escribiendo algo y tienes Twitter abierto y lees algo, pues... Al final terminas mirando la otra noticia".

Otra de las respuestas que dieron en relación a la lectura digital fue que en los medios digitales la portada se recarga con un volumen de noticias difícil de digerir. Para llegar a esta conclusión, se pidió a los informantes que mencionasen el número de noticias que componen la portada de un periódico digital y luego que indicasen cuántas se llegaban a leer. Todos reconocieron leer menos de diez del total de noticias que ven a diario en la portada, que puede oscilar, según el medio digital, de entre 30 a 40 artículos. En relación a esto último, algunos matizaron que las cinco primeras líneas que conforman una noticia en formato móvil bastan para mantenerse informados. P05:"Estamos todo el día enganchados al teléfono y nos es más fácil leer una noticia pequeña para saber qué ha pasado en Sudáfrica. $Y$ ya con cinco líneas me he enterado de lo que ha pasado. No necesito más", precisaba.

\subsection{Aproximación al discurso: relaciones categóricas}

La clasificación y análisis posterior de los datos permitió establecer una primera conexión entre las categorías extraídas de los resultados. 


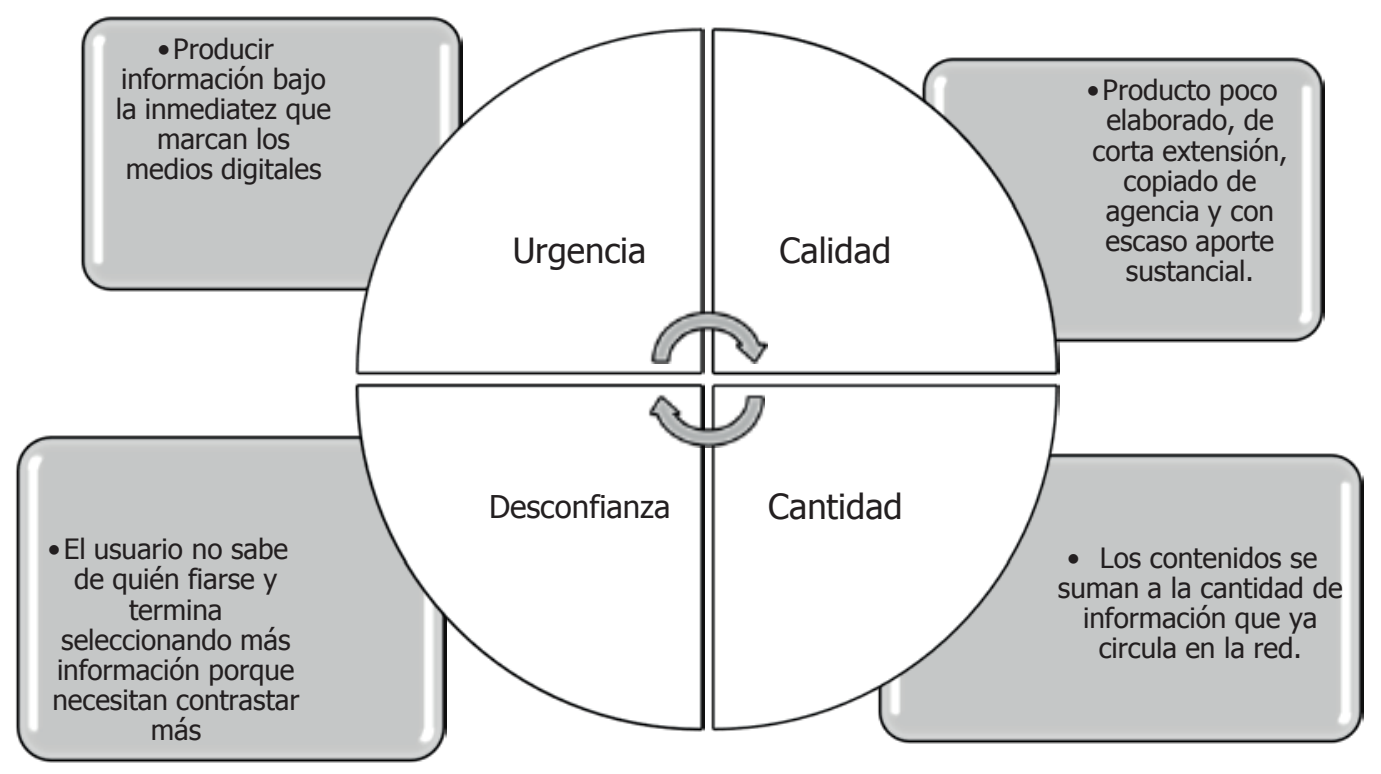

Figura 1: Relación causal entre categorías para explicar la aparición de infoxicación Fuente: Grupo de Discusión. Madrid.

La cantidad de información que hay en internet, así a primera vista, es identificada por los sujetos como una ventaja, ya que a más cantidad, mayor posibilidad de contrastar la información, por lo que uno tiene la posibilidad de informarse más y mejor. La teoría está clara. Ahora bien, los informantes mostraban signos de desconfianza muy elevados en sus expresiones. Esto que en un primer momento llegó a identificarse como subcategoría se convierte en uno de los ejes centrales del discurso cuando se advierte la siguiente asociación:

1. La urgencia que impera en los medios digitales no ayuda a reducir la infoxicación, sino más bien al contrario, con la publicación de información escueta y copiada, que a veces presenta errores e incoherencias.

2. Esta situación conduce a una pérdida de calidad, ofreciendo a los lectores contenidos fragmentados y superficiales, ya sea mediante una actualización a modo de timeline o con una previa de agencia que, por la falta de tiempo, no se amplia.

3. Si se parte de la premisa de que en internet existen tantos contenidos como uno quiera encontrar, el proceso de búsqueda y selección se complica aún más con este tipo de contenidos publicados, además por los propios medios de comunicación, que su cometido principal en este contexto de sobreabundancia es restar incertidumbre y clarificar la verdad.

4. Y si a esto se le añade que la posibilidad de que en internet cualquiera puede tener una blog y escribir artículos con apariencia noticiosa, sea el fin que tenga, la incertidumbre que genera en el sujeto es mayor. Esa misma desconfianza le hará extremar su criterio de contraste y el nivel de información que escogerá para analizar será notablemente superior. Por lo 
tanto, estamos ante un círculo vicioso en el que encontrar 'la aguja en un pajar' se convierte cada vez más en una complicada tarea, que posibilita la aparición de saturación informativa, ocasionando insatisfacción y agobio en el sujeto que la percibe.

\subsection{Discurso emergente}

A partir del análisis e interpretación de los datos es posible describir la percepción que tienen los periodistas sobre el fenómeno de la sobreabundancia informativa. Las ideas extraídas se irán contrastando con teorías e investigaciones previas sobre el ámbito de estudio para ver si corroboran o refutan el discurso emergente.

El concepto de infoxicación no surge en el discurso hasta que se les pide a los informantes que expliquen cómo se sienten cuando intentan asimilar un gran volumen de datos. Todos coinciden en señalar inseguridad, insatisfacción, desconfianza, agobio, frustración e incluso bloqueo. Un estado de derrota que procede de intentar asimilar y procesar más información de la que puede el sistema nervioso central. Estos sentimientos descritos por los sujetos coinciden, en rasgos generales, conconceptos en los que se relaciona la infoxicación con una patología, como el Síndrome de fatiga informativa (Lewis, 1996)o la Angustia informativa definida por Richard Wurman(2001).

Como se verá a continuación, la infoxicación puede interferir en cualquiera de las fases que conforman el proceso informativo, afectando tantoal rol lector comoal de productor de contenidos. 


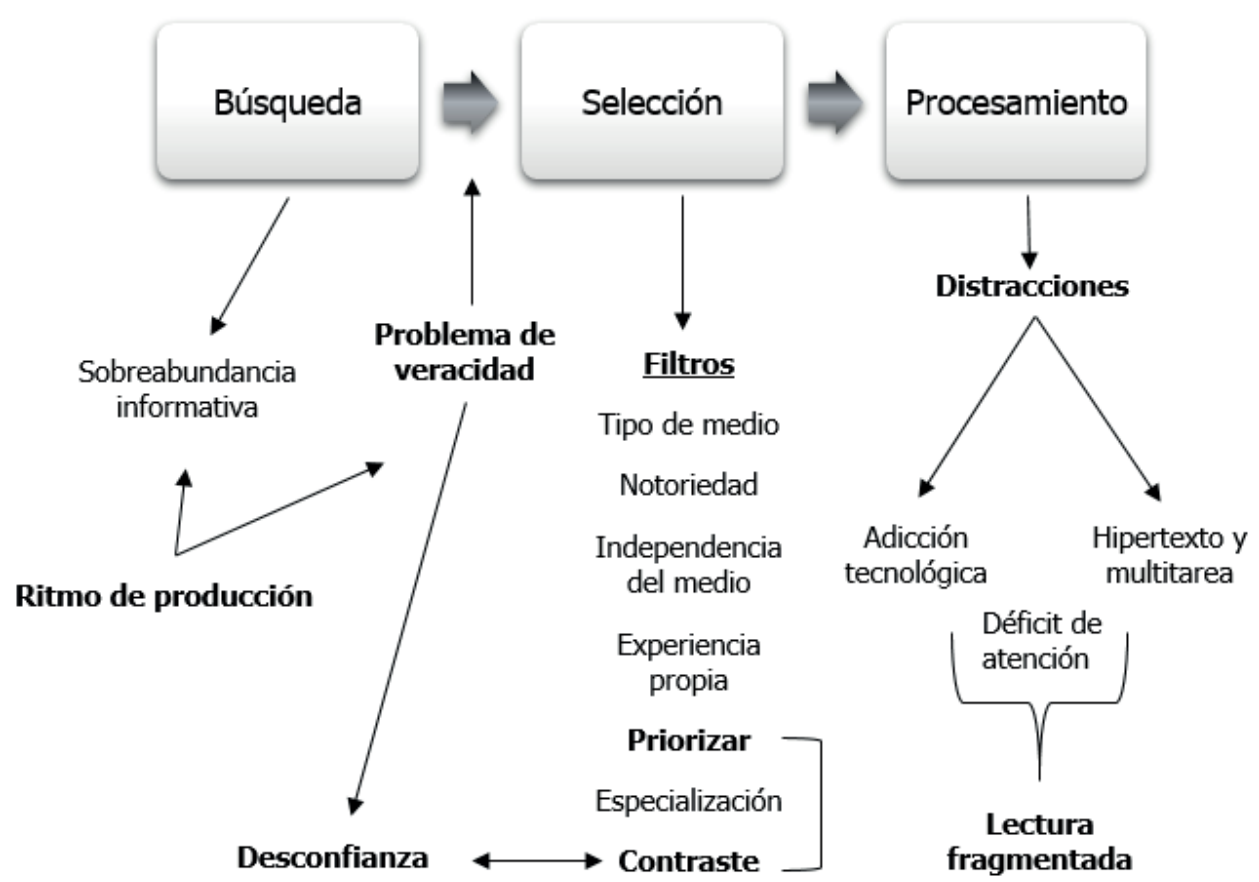

Figura 2:Diagrama explicativo de la infoxicación en relación con sus variables. Fuente: Grupo de Discusión. Madrid.

\subsubsection{Producción, volumen y veracidad de la información}

La infoxicación se puede experimentar durante el proceso de búsqueda por la cantidad de información disponible que hay en la red, pero en relación con el problema de veracidad de los contenidos, del que se habla continuamente en la discusión y que se alimenta de dos percepciones negativas que influirán en que el sujeto termine percibiendo infoxicación: inseguridad y desconfianza. Detrás de esta suposición hay una explicación teórica que tiene que ver con el ritmo de producción en internet. Cuanta más información, más libertad para escoger y más incertidumbre. Esta hipótesis ya ha sido postulada por Alfons Cornella (fundador del neologismo infoxicación), a la que denominó como Regla de las tres Y. Los usuarios tienen ahora más acceso a la información que antes, pero a la vez aumenta la falta de credibilidad de la fuente debido a la sobreabundancia, la multiplicidad de contenidos, el periodismo ciudadano, las difamaciones y otros contenidos de carácter comercial que se difunden bajo apariencia noticiosa y que ponen en riesgo el rigor periodístico. Como resultado, los lectores se informan con cierta sensación de incertidumbre (Cornella, Infonomia, 2011).

Otro elemento asociado a lasaturación informativa, y relacionado también con el ritmo de producción -en cómo se genera y transmite la información digital- es el principio de inmediatez e instantaneidadque impera en los medios digitales. Trabajar 
bajo esos parámetros aminora el análisis, la comparación entre fuentes y la contextualización, que a su vez acrecienta la inseguridad y el agobio percibidos por el periodista. Ante esta situación, se valora más la rapidez que la calidad y gran parte del contenido difundido por los medios son meros duplicados de agencia con algún que otro retoque de edición. Esta apreciación se sitúa en la misma línea que la descrita por el semiólogo Ignacio Ramonet en su libro La explosión del periodismo(2011), en el que vincula la producción de contenidos en la red con una nueva forma de ejercer censura en regímenes democráticos: no prohibiendo, sino informando hasta la asfixia. Bajo una rapidez que lleva a los medios a cometer cada vez más errores, es difícil digerir la información y transformarla en conocimiento.

\subsubsection{Selección y filtrado: especialización y contraste de fuentes}

La saturación informativa puede manifestarse durante el proceso de selección y filtrado porque de la habilidad personal y de las estrategias que se tengan para gestionar la información disponible dependerá el nivel de infoxicación consiguiente. La dieta informativa de los periodistas es amplia, y sus herramientas de selección y filtrado van desde la búsqueda en medios especializados que tengan cierta autoridad a motores de búsqueda concretos o seguimiento de temas por sindicación hasta listas de favoritos en Twitter y pestañas guardadas en el navegador, según detallaron durante la discusión.

Pero nopor ello ignoran los problemas que se generan al pretender estar informado de todo. De hecho, es la falta de criterio propio y la necesidad de querer saber todo de todo lo que conduce a la pérdida de control de información y a una dificultad para procesarla correctamente por su propia extensión. Esta idea de infoxicación como resultado de una carencia de adiestramiento y aprovechamiento de la tecnología para distinguir lo útil de lo superfluo se asemeja a la visión de Enrique Dans, quien no considera la infoxicación como un problema de cantidad, sino de adaptación (Dans), (Dueñas, 2010).

Así las cosas, los periodistas buscan en la especialización la fórmula para evitar la infoxicación, o al menos para no sentirse culpables por no abarcar más información. Sobre esto, Alfons Cornella explicaba en su artículo Cómo sobrevivir a la infoxicación(2000)que si una persona no es capaz de identificar cuáles son sus cinco temas prioritarios, el sujeto experimentará infoxicación inevitablemente porque no gestiona de modo eficiente la información y seleccionará más de lo que realmente va a poder abarcar.

En un contexto de saturación informativa, donde uno de los criterios a seguir para los periodistas es ponerlo todo en duda, los sujetos aseguran extremar la comparación y el contraste de fuentes. Sin embargo, se observa en los sujetos cómo 
esa propia desconfianza ocasionada por la sobreabundancia termina arrastrándoles al maremágnum informativo, y en lugar de conocer más sobre un tema, se produce el efecto contrario, acompañado de incertidumbre e insatisfacción. El término 'ignorante informado' que utiliza el documentalista Jorge Franganillo(2010) para referirse a este fenómeno viene a reforzar esta visión casi unánime de los entrevistados.

Como la desconfianza que se tiene es cada vez mayor en internet, a veces como solución, que no ayuda precisamente, el sujeto tiende a buscar información que refuerce sus convicciones. Esta práctica, incorrecta para informarse, se identificó con la siguiente expresión que aportó uno de los participantes: "Ahora hay más, más medios, y puedes ir contrastando en cada uno de ellos para ir buscando la información que tú te crees" (P06). Algo que guarda cierta similitud con la interpretación recogida de la Teoría de la disonancia cognitiva, que incide en que el desequilibro ocasionado entre las opiniones y la información se resuelve con elementos cognoscitivos consonantes. Es decir, con información que refuerce la opinión inicial del sujeto (Ovejero Bernal, 1993).

\subsubsection{Procesamiento de información interrumpido}

Por último, la infoxicación puede aparecer durante la recepción y procesamiento de la información por las distracciones. Según el análisis de los datos obtenidos, éstas pueden ser originadas por:

1. Adicción tecnológica.El primer aspecto relacionado con las distracciones deriva de la integración de internet y las nuevas tecnologías en la vida diaria de los sujetos. En concreto, la conectividad continuada, las redes sociales y los sistemas de mensajería instantánea a través de dispositivos móviles, que llevan al usuario a hacer un uso impulsivo y compulsivo del mismo. En este sentido, el Síndrome de nomofobia (The App Date, 2013) y otros estudios de dependencia sobre el uso de lasTIC presentados en el artículo se verían reforzados con el discurso,planteandoestos hábitos de consumo como una amenaza real para la concentración y el auto control del uso de internet.

2. Navegación hipertextual y multitarea. La navegación diagonal y la posibilidad de tener varias ventanas abiertas en el navegador estáalterando el modelo tradicional de lectura. Esto viene a reforzar la teoría del hipertexto (Díaz Noci, 2004), ya que de los datos obtenidos se observa una tendencia general a practicar el multitaskingen la red.

En consecuencia, estas distracciones convierten la atención en un recurso escaso y se evidencia unaeconomía de la atención. Es decir, que disminuye el tiempo que se 
tiene para atender a los estímulos entrantes(Goldhaber, 2006), a la vez que el sujeto sufre déficit de atención en la memoria de trabajo(Baddeley \& Logie, 1999): dificultad para controlar selectivamente la atención y recordar las instrucciones prioritarias, porque éstas se verán interrumpidas continuamente por nuevos estímulos. Teorías que coinciden también con la descripción de limitación atencional dada por el arquitecto Richard Wurman (2001).De esta forma, el sujeto intentará prestar atención a varios estímulos a la vez, de forma corta e interrumpida, para dirigirla posteriormente a otros, lo que incita a una lectura superficial y fragmentada, tal y como vaticinabanNicholas Carr (2010), García y Gertrudix (2009) y Raúl Trejo Delarbre(2001). Como consecuencia, el sujeto experimenta un estado de derrota frustración y angustia-, por lo que el pensamiento crítico sí puede verse perjudicado. Esta suposición se vería reforzada poraportaciones extraídas del grupo de discusión como esta: "De tener poco tiempo e intentar leer varias cosas en ese tiempo y, al final, te das cuenta de que no puedes y te bloqueas" ( $\mathrm{PO} 3$ ).

Si bien es cierto que esta apreciación no puedegeneralizarse ni extrapolarse más allá del segmento poblacional del estudio, también lo es que el lector de un medio digital tiende a hacer un escáner para identificar rápidamente lo que le interesa leer y luego termina en otras noticias destacadas del día. Por tanto, se corrobora que se está produciendo un cambio en los hábitos de lecturaque, junto a la creencia generalizada de poder ejecutar varias tareas a la vez, las distracciones y la falta de un criterio de selección riguroso, sí que podría repercutir negativamente en elpensamiento crítico de los sujetos. En grandes rasgos, la teoría guarda cierta similitud con informes realizados recientemente por la plataforma de neurocientíficos y psicólogos europeos E-Read, que pone de manifiesto los efectos cognitivos que provocan los nuevos formatos. (COST: European Cooperation In Science And Technology, 2014).

\section{CONCLUSIONES}

Los resultados obtenidos permiten alcanzar las siguientes conclusiones:

- La infoxicación es un problema de cantidad de información, pero en relación con el problema de la veracidad y la desconfianza que perciben los sujetos ante los contenidos que circulan por internet, incluso los creados por los propios periodistas.

- También es un problema de orden social por el desconocimiento de las herramientas y recursos que ofrece la propia tecnología (diseño de información, RSS, curadores de contenido para organizar y hacer seguimiento de temas concretos) y la falta de criterios de selección, porque de no emplearse y, por tanto, no priorizar y descartar los estímulos entrantes, el volumen de datos seleccionados para procesar rebosará la propia capacidad cerebral para atenderlos. 
- Los periodistas se encuentran ante un dilema entre lo que desean saber (ya sea por su especialización o interés individual) y la premisa impuesta a los periodistas de "saber un poco de todo". Es preferible estar actualizado de uno o dos temas para tener un conocimiento mayor y más amplio que 'picotear' un poco de todo y percibir insatisfacción al no poder abarcarlo todo, lo que lleva a experimentar saturación.

- La adicción tecnológica, sobre todo la procedente del teléfono móvil, genera problemas relacionados con la atención y el procesamiento de la información. Es necesario incrementar el control que hacemos del móvil para evitar distracciones.

- La navegación hipertextual y la multitarea también distraen la mente. Generan un déficit de atención, y debilitan la lectura y el pensamiento crítico. Para evitarlo, es necesario que se haga una lectura lineal y completa del texto y organizar la mente siguiendo un plan de prioridades, como puede ser planificar las tareas que se quieren llevar a cabo y dividirlas en bloques de tiempo para evitar el colapso informativo ocasionado por la ejecución simultánea de tareas.

- Estar desbordados de información no equivale a poder ni a conocimiento. Vale más la calidad que la cantidad. Esta idea debería calar en el tejido de las empresas periodísticas para renunciar al modelo de la inmediatez, cuya prioridad es saturar la portada con noticias las 24 horas del día, independientemente de que sea un teletipo de agencia (con una edición mínima y dando por sobrentendida la veracidad del contenido sin revisar previamente las fuentes). De momento, la instantaneidad de los contenidos más ligados a la actualidad seguirá siendo un riesgo de infoxicación, pues estas noticias, junto a otras orientadas a aumentar el número de páginas vistas, basadas en tendencias de Twitter y temas sensacionalistas, debilitan la confianza de los lectores y generan más confusión.

En definitiva, no es cuestión de implementar controles en internet, que es libre por su propia naturaleza y además atentaría contra la libertad de expresión. Tampoco es cuestión de reducir o limitar el ritmo de producción, ya que su avance supera al propio desarrollo de herramientas tecnológicas que faciliten trabajar sobre estos datos. Pero sí hace falta reivindicar la ética profesional, recordando que en un mundo de sobreabundancia, lo que el ciudadano necesita es información trascendente, contextualizada e interpretada por periodistas, que otorguen un valor al contenido que lo diferencie del ruido que circula por internet $y$, en consecuencia, que ayude al lector a comprender el mundo en el que vive para que pueda transformar su juicio en conocimiento. El periodismo tiene esa tarea encomendada en la sociedad y no ha variado por el hecho de que los medios de comunicación sean ahora digitales, por lo que la apresurada producción informativa no puede ir en detrimento de la calidad y la búsqueda de la verdad. 


\section{REFERENCIAS}

APM. (2014). Informe Anual de la Profesión Periodística 2014. Madrid.

AxiCom y Top Comunicación. (2013). La Función de comunicación vista por los profesionales de la información.

Baddeley, A. D., \& Logie, R. H. (1999). Working Memory: The Multiple-Component Model. En P. Shah, Models of working memory: Mechanisms of active maintenance and executive control (págs. 28-61). Nueva York: Akira MIyake's.

Ballesteros Guerra, J. C., \& Megías Quirós, I. (2015). Jóvenes en la red: un selfie. Obtenido de http://adolescenciayjuventud.org/images/pdf/selfie-resumenejecutivo.pdf

Berg, B., \& Lune, H. (2004). Qualitative research methods for the social sciences. Boston: Pearson.

Carr, N. (2010). Superficiales. ¿Qué está haciendo Internet con nuestras mentes? Bogotá: Taurus.

Castells, M. (1998). La era de la información. Alianza Editorial.

Castells, M. (2000). Internet y la Sociedad Red. Lección inaugural del programa de doctorado sobre la sociedad de la información y el conocimiento. Barcelona.

CIS. (2015). Barómetro de marzo. Obtenido de http://datos.cis.es/pdf/Es3057mar_A.pdf

Cornella, A. (1999). A mayor desarrollo informacional, menor infoxicacion. El profesional de la información. Obtenido de http://www.elprofesionaldelainformacion.com/contenidos/1999/septiembre/a_ mayor_desarrollo_informacional_menor_infoxicacion.html

Cornella, A. (2000). Cómo sobrevivir a la infoxicación . Acto de entrega de títulos de los programas de Formación de Posgrado del año académico 1999-2000.

Cornella, A. (25 de Marzo de 2011). Infonomia. Obtenido de 'Infoxicación': http://www.infonomia.com/articulo/ideas/7150

COST: European Cooperation In Science And Technology. (2014). Evolution of reading in the age of digitisation. Bruselas. 
Díaz Noci, J. (2004). Teoría de los géneros ciberperiodísticos. II Congreso Iberoamericano de Periodismo Digital. Santiago de Compostela.

DOMO. (2014). Data never sleeps 2.0.

Dueñas, J. (12 de 10 de 2010). Infoxicación: un 'mal' necesario. El Tiempo.

Franganillo, J. (5 de 04 de 2010). La ansiedad informativa. Diario Uno.

García García, F., \& Gértrudix Barrio, M. (2012). El Mare Nostrum digital: Mito, ideología y realidad de un imaginario sociotécnico. Revista ICONO14. Revista CientíFica De ComunicacióN Y TecnologíAs Emergentes, 7(1), 7-30. doi:http://dx.doi.org/10.7195/ri14.v7i1.331

Goldhaber, M. H. (2006). The value of openness in an attention economy. First Monday.

Harris, M. (1990). Antropología Cultural. Madrid: Alianza Editorial.

Hilbert, M., \& López, P. (2011). The World's Technological Capacity to Store, Communicate and Compute Information. Nueva York: Science Express.

INE. (2014). Encuesta sobre Equipamiento y Uso de Tecnologías de Información y Comunicación en los Hogares.

Johnson, C. A. (2011). The Information Diet: A Case for Conscious Consumption. O'Reilly Media.

Kirsh, D. (2000). A few thoughts on Cognitive Overload. Intellectica, 19-51.

Klingberg, T. (2009). The Overflowing Brain: Information Overload and the Limits of Working Memory. Oxford University Press.

Lewis, D. (1996). Dying for Information? Londrés: Reuters Business Information.

Lyman, P., \& Varian, H. R. (2003). How Much Information? Berkeley: University of California at Berkeley. Obtenido de https://www.ischool.utexas.edu/ i385e/readings/how-much-info.pdf

McCombs, M. (1996). Los efectos de los medios de comunicación. Investigaciones y teorías. Barcelona: Paidos.

McLuhan, M. (1993). La galaxia Gutenberg. Barcelona: Galaxia Gutenberg. 
Mella, O. (2000). Grupos Focales: Técnica de investigación cualitativa. Santiago: CIDE.

Meyer, P. (1993). Periodismo de precisión. S.A. BOSCH.

Miller, G. (1956). El mágico número 7 más o menos 2.

Nielsen. (2014). So Many Apps, So Much More Time for Entertainment.

Nielsen. (2015). El mundo digital y el impacto de las nuevas formas de consumo audiovisual.

O'Reilly, T. (2006). Web 2.0 Principles and Best Practices. O'Reilly Radar. Obtenido de http://oreilly.com/catalog/web2report/chapter/web20_report_excerpt.pdf

Ovejero Bernal, A. (1993). Teoría de la disonancia cognoscitiva. Psicothema. Obtenido de http://www.unioviedo.net/reunido/index.php/PST/article/download/7156/7020

Posner, M., \& Petersen, S. (1990). The attention system of the human brain. Annual Review of Neuroscience.

Postman, N. (1998). Five Things We Need to Know About Technological Change. The New Technologies and the Human Person: Communicating the Faith in the New Millennium (págs. 3-7). Denver Colorado: cGraw-Hill.

Prensky, M. (2010). Nativos e inmigrantes digitales. Institución SEK.

Ramonet, I. (1999). El periodismo del nuevo siglo. La Factoría. Obtenido de http://www.revistalafactoria.eu/articulo.php?id=115

Ramonet, I. (2011). La explosión del periodismo. Madrid: Clave Intelectual.

Small, G. W. (2009). Your Brain on Google: Patterns of Cerebral. The American Journal of Geriatric Psychiatry.

Strauss, A. y. (2002). Bases de la investigación cualitativa: técnicas y procedimientos para desarrollar la teoría fundamentad. Medellín: Universidad de Antioquia.

The App Date. (2013). Informe APPS Septiembre 2013. Madrid. Obtenido de http://madrid.theappdate.com/informe-apps-2013/

Toffler, A. (1973). El shock del futuro. Esplugas de Llobregat, Barcelona: PLAZA\&JANES, S.A. 
Trejo Delabre, R. (2006). Viviendo en El Aleph. La sociedad de la información y sus laberintos. Barcelona: Gedisa.

Trejo Delarbre, R. (2001). Vivir en la Sociedad de la Información. Revista Iberoamericana de Ciencia, Tecnología y Sociedad.

White, D. M. (1950). The gate keeper: $A$ case study in the selection of news. Obtenido de http://www.aejmc.org/home/wpcontent/uploads/2012/09/Journalism-Quarterly-1950-White-383-90.pdf

Winner, L. (1979). Tecnología autónoma: la técnica incontrolada como objeto del pensamiento político. Barcelona: Gustavo Gili.

Wurman, R. S. (2001). Angustia informativa. Buenos Aires, Argentina: Pearson Education S.A.

\section{Ruth Franco Rodríguez}

Graduada en Periodismo por la Universidad Rey Juan Carlos de Madrid, y colaboradora del Grupo de investigación Ciberimaginario de la Universidad Rey Juan Carlos. Autora de la investigación "Sobreabundancia informativa y economía de la atención".

\section{Manuel Gértrudix Barrio}

Profesor Titular de Comunicación Digital de la Universidad Rey Juan Carlos, e Investigador Principal del Grupo de investigación Ciberimaginario (www.ciberimaginario.es) 